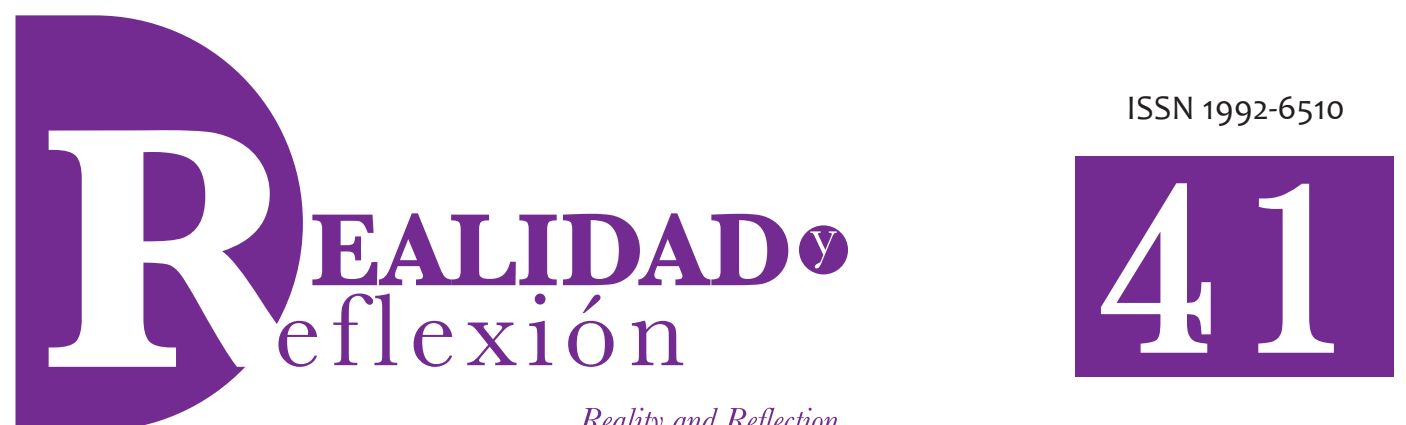

Reality and Reflection

año 15, N 41, San Salvador, El Salvador, Centroamérica Revista Semestral Enero-Junio 2015

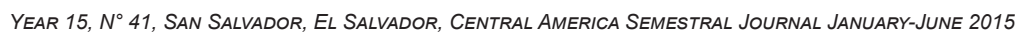

\title{
Capital social, sinergia, impacto social y las organizaciones de la sociedad civil
}

\author{
Social capital, synergy, social impact and \\ civil society organizations
}

Francisco Álvarez

Doctor en Ingeniería en Sistemas

Universidad Nacional Autónoma de México

M.A. Ingeniería en Sistemas

Universidad Nacional Autónoma de México

\section{RESUMEN}

En la actualidad no es posible hablar de un criterio unificado en lo que a capital social, sinergia, y la medición de impactos sociales se refiere; más bien, la conceptualización y la identificación de las variables de interés dentro de un estudio para la valoración de los impactos generados, a través de proyectos de coinversión social, dependerá de las características del estudio (micro, meso, macro) y del objetivo-meta que se trace. CAPITAL SOCIAL, SINERGIA, IMPACTO SOCIAL.

\section{ABSTRACT}

Nowadays, we cannot speak about of unified approach as far as social capital and social impact assessment, rather the conceptualization and identification of relevant variables. In this way is possible establish that a study for assessing impacts, generated through social co-investment projects, depends mainly of the characteristics of the study (micro, meso, and macro) and the goals and purposes established at the begining of the project. SOCIAL CAPITAL, SYNERGY, SOCIAL IMPACT. SOCIAL CAPITAL, SINERGY, SOCIAL IMPACT. 
En la actualidad, y sobre todo en Latinoamérica, el desarrollo comunitario a partir del concepto del capital social ha cobrado gran importancia, no sólo por el concepto mismo, sino más bien por la potencialidad inmersa dentro de éste, en referencia a la búsqueda del desarrollo integral de comunidades carentes de los recursos y del capital (económico, político, intelectual, entre otros) necesario para alcanzar un bienestar social que les permita allegarse de mejores condiciones de vida basadas en la confianza, la sinergia, la cooperación y el respeto, dentro de un intricado marco de redes sociales capaces de buscar un beneficio colectivo, en donde la sociedad civil organizada juega un papel preponderante para el desarrollo de proyectos orientados para tal fin.

\section{El capital social}

A lo largo de los últimos 25 años el concepto de capital social ha venido evolucionado de forma importante, tanto en el campo teórico como en el práctico (dentro de diversas disciplinas y medios). El marco de referencia inmerso dentro del capital social permite en mayor o menor medida dilucidar de una mejor manera la forma en que los entes sociales interactúan para la consecución de beneficios individuales y colectivos (Brunie, 2009 y Claridge, 2007). Por lo anterior, el capital social ha sido empíricamente vinculado de forma positiva con posibles resultados favorables en lo que se refiere a cambio climático (Adger, 2003), vida comunitaria (Krishna, 2002), democracia y Gobierno (Uslaner, 2003), manejo de desastres (Murphy et al, 2005), desarrollo económico (Krishna, 2002 y 2008) administración y conservación de recursos naturales (Ostrom, 2000) y educación, trabajo y organización (Zhao, 2002) entre otros. A pesar de ello, actualmente no existe una definición consensuada sobre lo que se entiende por capital social. En este contexto, Bourdieu (1986), Coleman (1988, 1990), Evans (1996) y Putnam (1993, 1995, 2000 y 2001) resultan ser los exponentes más connotados dentro del campo de la teorización del capital social, por lo que para entender el concepto adecuadamente se hace necesario realizar una breve revisión de las ideas expuestas por dichos autores.

\subsection{El capital social de Bourdieu}

Pierre Bourdieu (1986), sociólogo europeo, desarrolló el concepto del capital social sobre la base de cómo las clases sociales y otras formas de iniquidad son socialmente reproducidas (Capriano, 2006 y Field, 2003) y definió el capital social como los recursos agregados, actuales o potenciales, vinculados a la posesión o existencia de una red de trabajo, a largo plazo, poco más o menos institucionalizada, basada en el reconocimiento y en la relación entre individuos. Para Bourdieu (1986), el capital social puede ser visto a través de las características de los grupos sociales, considerando la existencia o la cantidad de los recursos ligados a una red social de trabajo institucionalizada dada. Los beneficios obtenidos por los integrantes de la red social sirven como base para la construcción de los lazos de solidaridad, que promueven nuevamente la consecución de nuevos beneficios.

De igual manera, Bourdieu identifica otras formas de capital (cultural educacional, entre otros) con una alto grado crítico de importancia para la obtención de beneficios y el mantenimiento del orden social, los 
cuales pueden ser utilizados en tándem en ausencia del capital financiero. Así mismo, el capital social depende sobre todo de dos factores: (1) La dimensión de las conexiones de la red que permite movilizar, y la movilización del individuo; y (2) La cantidad y el tipo de capital que posee el individuo.

La teoría desarrollada por Bourdieu (Capriano, 2006) impele a considerar no solamente la existencia de una red comunitaria social y la cantidad de los recursos (potenciales o actuales) en posesión de la red; sino que también las destrezas hacia el interior de la comunidad direccionan los recursos y habilidades de un colectivo, con el objeto de obtener beneficios a partir de un intercambio de bienes o servicios. Dentro de su teorización desarrolla ideas importantes sobre la forma en que opera el capital para beneficio del grupo y la forma en que estos obtienen los recursos y el poder, reconociéndose algunos posibles aspectos sociales negativos, como la exclusión de los beneficios ligados a la red, de algunos individuos dentro del sistema.

\subsection{El capital social de Coleman}

Para Coleman (1988 y 1990) el capital social constituye un recurso cuya particularidad radica en ser algo inherente a la estructura de las relaciones sociales (Forni et al, 2004). Este recurso facilita el logro de objetivos personales que no podrían alcanzarse de una forma individual o porque el costo y los recursos a comprometerse en la consecución del objetivo serían demasiado altos o cuantiosos. Es así como dentro de su concepción teórica, Coleman concibe el capital humano en relación a la utilización de individuos próximos o conocidos (dentro de una red) para la consecución de objetivos y la reinversión del capital obtenido para la obtención de más y mejores beneficios, bajo un sistema basado en obligaciones, expectativas, sanciones, normas $y$ responsabilidades. Cabe destacar que dicho sistema se encuentra condicionado por (1) El grado de confiabilidad del entorno social; (2) Las necesidades presentes en los individuos; y (3) El grado de cercanía e interrelación dentro de la red social.

En esta dirección Coleman establece que el capital social, como bien público requiere de una regulación interna que norme la actividad y la conducta de los integrantes de la red, con el objeto de prevenir y sancionar el surgimiento de conductas indebidas que puedan poner en riesgo la estabilidad del sistema. De igual forma en dicho enfoque se deja entrever que los resultados obtenidos por una red social, a través de uno o más miembros, permiten de una forma o de otra, que individuos no ligados a dicho esfuerzo pero sí inmiscuidos dentro del sistema o tejido social, gocen en mayor o menor medida de los beneficios obtenidos por la actividad del colectivo.

\subsection{El capital social de Putnam}

Putnam define el capital social (Capriano, 2006) como aquellas características inherentes de una organización social, tal como las redes sociales, las normas y la confianza social, las cuales facilitan la coordinación y la cooperación para beneficio mutuo. Así mismo, Putnam plantea que la cantidad de capital social presente en una comunidad (pueblo, municipio, etc.) conforma características colectivas generadas vía costumbres, normas, reciprocidad y confianza entre los residentes; dichas relaciones poseen 
implicaciones importantes en la consecución de bienes/servicios, las cuales se traducen en beneficios a los integrantes de la red social, mediante un compromiso cívico.

En este sentido, dentro del Capital Social, Coleman establece que las conexiones y las diversas formas de éstas, entre los individuos e instituciones, representan un punto clave y a la vez crítico en la formación y el desarrollo de redes de cooperación, en donde el compromiso cívico, la igualdad política, la solidaridad-confianza-tolerancia y la vitalidad asociativa forman los pilares sobre los que se basan o se debe basar cualquier red colaborativa dentro de un sistema social dado.

\subsection{El capital social de Evans}

Evans (1996) sugiere que el capital social es formado a través de un actor que pertenece más al aparato del Estado que a la sociedad en donde trabaja, dando lugar a la creación de sinergias entre los ciudadanos y los agentes públicos, permitiendo alcanzar metas y objetivos mucho más amplios que de manera individual (Wakefield et al, 2005). Bajo esta concepción el factor clave no radica en la conexión per se, si no en el escalamiento a un nivel mucho mayor, de los enlaces o vínculos desde las organizaciones, para que éstas y los resultados en vías de obtención sean política y económicamente efectivos y viables.

De acuerdo a lo anterior, el rol del Estado y las organizaciones dentro del capital social se enfatizan y se destaca que la robustez, la sofisticación y la dotación de los recursos necesarios (en referencia a las instituciones públicas) son un desarrollo de y desde el capital social, en donde existe una responsabilidad de las instituciones públicas en transferir o trasladar los vínculos o enlaces sociales dentro del desarrollo de las comunidades.

\section{Diferentes tipos de capitales sociales}

Dentro del capital social se ven involucrados ciertos factores como: (1) La cohesión social; (2) Las diversas clases, formas y modos de capital; (3) Los controles formales e informales dentro de la operación y normalización de conductas y gobernanza de las acciones sociales; (4) Los recursos reales y potenciales de la red social; (5) Los tipos de interacción social; (6) Las relaciones de confianza, solidaridad $y$ tolerancia; y finalmente, (7) La participación y la organización de la comunidad y de los individuos involucrados (Brunie, 2009).

Los factores antes mencionados cobran importancia en la práctica, ya que estos influyen y/o garantizan en mayor o menor medida la generación de beneficios tangibles y/o intangibles dentro de las redes sociales y comunidades. Más que hablar de diversas teorías es posible hablar de diferentes tipos de capitales sociales que coexisten de manera cotidiana y hasta cierto punto se entremezclan en el quehacer diario de las organizaciones y las comunidades sociales.

El capital social es un concepto complejo que incluye diversas variables y factores, que pueden presentarse de diversas formas, dependiendo de la envergadura de las interacciones en que éste se desarrolle. Brunie (2009) identifica al menos tres perspectivas (clústeres) desde las cuales puede analizarse el capital social: (1) La perspectiva relacional; (2) La perspectiva colectiva; y (3) La perspectiva generalizada. 
Dichas perspectivas varían de acuerdo al alcance del nivel de manifestación dentro de la vida social, la dimensión que enfatiza, la parte central del proceso, la utilidad y la fungibilidad a través del campo de acción o dominio (Cuadro 1).

\subsection{El modelo relacional}

La perspectiva relacional (Brunie, 2009) es fundamentalmente la habilidad de los actores para movilizar sus contactos sociales en orden de la obtención de recursos. En este caso, el capital social relacional se encuentra ligado a los actores de la red social, sean estos individuos $u$ organizaciones. Esta conceptualización se enfoca sobre las relaciones que los actores desarrollan y mantienen con otros, reconociendo dentro de esta relación dos factores de importancia: (1) La cantidad y la calidad de los recursos disponibles en el entorno interpersonal de los actores; y (2) Las relaciones sociales que proveen el acceso a esos recursos (Portes, 1998).

El capital social relacional es entonces el producto de la inversión intencional dentro de las relaciones entre individuos o grupos, y su utilidad radica en el acceso diferencial a los recursos que son proveídos a los actores en cuestión. Es así que el capital social relacional puede ser observado a nivel micro (individual) o a nivel meso (cuando se observan relaciones entre grupos y organizaciones). Bajo este entendido, el capital social puede proveer beneficios individuales, pero también puede facilitar el funcionamiento de la vida social y política o el apalancamiento del beneficio social (Van der Gaag et al, 2004). Es entonces que desde la perspectiva relacional, diferentes tipos de recursos son evaluados desde distintos propósitos y el valor de las relaciones sociales de los actores depende del tipo de los recursos buscados. Bajo esta circunstancias el capital social es descrito en términos de redes sociales vistas bajo dos dimensiones importantes: (1) Los recursos de la red social -en cuyo análisis se examina la estructura de la red en situaciones rutinarias y los recursos embebidos dentro de ésta-; y (2) La estructura de la red social, -cuyo estudio se relaciona con el análisis con la movilización del capital que se encuentra basado en los recursos activados en pos de un beneficio específico y sobre la naturaleza de las vinculaciones activadas-.

En la actualidad, se han presentado dos desafíos importantes dentro de la perspectiva del capital social relacional: (1) El balancear el análisis de la red de recursos y la estructura de la red; y (2) El seguimiento entre el acceso y la movilización del capital social. Estos dos desafíos en la práctica se observan en estudios realizados dentro del campo del capital social relacional, los cuales pueden ser clasificados de acuerdo al tipo de análisis realizado de acuerdo al énfasis diferencial establecido en los recursos de la red y su estructura, identificando primordialmente dos grandes categorías: (1) Los estudios enfocados principalmente sobre el estudio de los recursos sociales; y (2) Los estudios sobre redes sociales de trabajo (Hurlber et al, 2000 y Lin, 2008).

El capital social colectivo está basado en la densidad de las interacciones, donde la confianza mutua es el factor central en la facilitación de la cooperación voluntaria, la cual surge a partir de normas de reciprocidad y redes de trabajo social. Dichas normas de 


\section{Cuadro 1}

\section{Resumen de las diferencias conceptuales entre las tres perspectivas desde las que se puede considerar el capital social.}

\begin{tabular}{|c|c|c|c|}
\hline & Perspectiva relacional & Perspectiva colectiva & Perspectiva garantizada \\
\hline $\begin{array}{l}\text { Nivel de manifestación } \\
\text { dentro de la vida social }\end{array}$ & $\begin{array}{l}\text { Redes de grupos } \\
\text { individuales u } \\
\text { organizaciones. }\end{array}$ & $\begin{array}{l}\text { Pequeño, relativamente } \\
\text { homogéneo y en grupos } \\
\text { exclusivos. La calidad de } \\
\text { las relaciones entre los } \\
\text { miembros del grupo. }\end{array}$ & $\begin{array}{l}\text { El capital social es un } \\
\text { atributo individual. }\end{array}$ \\
\hline Dimensión que enfatiza & $\begin{array}{l}\text { Recursos embebidos en } \\
\text { redes sociales personales, } \\
\text { las cuales proveen los } \\
\text { recursos. }\end{array}$ & $\begin{array}{l}\text { La densidad de las } \\
\text { interacciones. }\end{array}$ & $\begin{array}{l}\text { Actitudes individuales y } \\
\text { predisposiciones hacia } \\
\text { otros y a veces normas } \\
\text { sociales institucionales. }\end{array}$ \\
\hline $\begin{array}{l}\text { Núcleo central del } \\
\text { proceso }\end{array}$ & $\begin{array}{c}\text { Acceso y habilidad } \\
\text { diferencial para movilizar } \\
\text { los recursos valorados vía } \\
\text { movilización de contactos } \\
\text { sociales. }\end{array}$ & $\begin{array}{l}\text { El capital social se define } \\
\text { en relación de su función } \\
\text { como factores que } \\
\text { facilitan la cooperación: } \\
\text { redes de trabajo, normas y } \\
\text { reciprocidad y la fortaleza } \\
\text { de la confianza. }\end{array}$ & $\begin{array}{l}\text { Confianza generalizada } \\
\text { que influye en la forma } \\
\text { en que las personas se } \\
\text { relacionan entre sí. }\end{array}$ \\
\hline Utilidad & $\begin{array}{l}\text { Actividades primarias } \\
\text { para beneficio de } \\
\text { actores (individuales o } \\
\text { corporativos). }\end{array}$ & $\begin{array}{l}\text { Actividades grupales } \\
\text { específicas que no } \\
\text { pueden ser valoradas } \\
\text { de forma individual } \\
\text { (acciones colectivas); } \\
\text { pero las características del } \\
\text { capital social permiten el } \\
\text { beneficio colectivo. }\end{array}$ & $\begin{array}{l}\text { Bienes sociales que unen } \\
\text { a la sociedad y promueven } \\
\text { un comportamiento cívico. }\end{array}$ \\
\hline $\begin{array}{c}\text { Fungibilidad a través } \\
\text { del campo de acción o } \\
\text { dominio }\end{array}$ & $\begin{array}{l}\text { Limitada, debido a } \\
\text { que el valor de uso } \\
\text { de los recursos y las } \\
\text { características de la red } \\
\text { son asuntos o cuestiones } \\
\text { específicas. }\end{array}$ & $\begin{array}{l}\text { Limitado a actividades que } \\
\text { requieren vías similares de } \\
\text { expectativas a las de una } \\
\text { organización. }\end{array}$ & Fungible. \\
\hline
\end{tabular}

Fuente: Adaptado de Brunie (2009).

reciprocidad predisponen a los individuos a la cooperación, restringiendo las conductas oportunistas y reforzando la confianza mutua dando a lugar que las redes de trabajo incrementen la cooperación debido a los bajos costos de transacción a través de las vías de interacción existentes y porque éstas facilitan la comunicación de la información hacia los demás actores, así como la detección de discrepancias y desavenencias. Cabe señalar que tanto la confianza y las redes de trabajo se ven afectadas por las reglas en uso.

Dentro de la perspectiva colectiva el capital social se compone de las redes de trabajo 
(reglas, precedentes y procedimientos inmersos en la red) y de las actitudes, conductas, reglas de comportamiento, valores compartidos, confianza y normas de reciprocidad generalmente aceptadas (Brunie, 2009). Bajo esta óptica el capital social requiere de la existencia de pequeños grupos exclusivos y relativamente homogéneos (Newton, 1997). Estos requerimientos son necesarios para permitir que la densidad de las interacciones sea la adecuada para soportar la estructura del capital social. Resulta importante destacar que este tipo de modelos es aplicado en pequeñas villas dentro del mundo desarrollado, grupos que cuentan con una variedad de recursos comunes, asociaciones vecinales, grupos comunitarios de vigilancia o comunidades compuestas por minorías, por lo que dicho esquema de capital social permite alcanzar e implementar de manera fácil y oportuna las decisiones colectivas en diversas áreas de importancia para la comunidad o red social en cuestión.

\subsection{El modelo generalizado}

Este modelo generalizado concibe al capital social como una propiedad subjetiva de los individuos, es decir, los valores y actitudes que influencianla manera en quelas personas se relacionan entre sí, los predispone a la cooperación, la confianza, el entendimiento y a la empatía entre ellas (Brunie, 2009). Es así como la confianza resulta ser la piedra angular del capital social generalizado (la reciprocidad generalizada, las normas de tolerancia, y en ocasiones la confianza en las instituciones son enfatizadas en la perspectiva generalizada).

El capital social generalizado subyace en el comportamiento y en las actitudes de individuos y por ende en sus atributos individuales. No es el grupo per se el que construye la confianza, sino más bien son los miembros del grupo los que adquieren una serie de valores y actitudes particulares. Toda vez que dichos valores y actitudes son interiorizados por el grupo, se incorporan y aplican de manera general a través de la red de trabajo, aplicándose en todas y cada una de las relaciones y transacciones entre los individuos, prevaleciendo de una forma o de otra la confianza entre cada una de las partes involucradas. Lo anterior permite no solamente el funcionamiento de una red social de forma generalizada, sino que igualmente conlleva a la creación de una conciencia social compartida, que incita a la promoción de la realización de acciones altruistas hacia otros individuos o grupos sociales, dando como resultado el involucramiento de los miembros de la red en causas caritativas, políticas, entre otras.

El enfoque de capital generalizado se ha aplicado principalmente en estudios dentro de los campos siguientes: (1) Estudios que conciben el capital social como la relación entre la participación y la confianza a un nivel individual (Shah, 1998); (2) Estudios donde se conserva la idea de que las interacciones sociales son el núcleo central del capital social, pero se encuentra en desacuerdo con la naturaleza de la relevancia de las interacciones (Hooghe, 2008); (3) Estudios que minimizan el rol de las redes de trabajo argumentando que el capital social es producido mediante las instituciones (Rothstein, 2008); y (4) Estudios de investigación sobre determinantes actitudinales del capital social, enfocados a relaciones en lo concerniente a valores, hábitos, disposición de individuos y confianza social. 


\subsection{Sinergias}

Dentro del capital social los componentes y elementos que lo conforman resultarían inútiles si no existiera un catalizador que permita una interacción efectiva entre cada uno de los individuos que componen una red social dada; y es justamente en este punto donde la sinergia (entre cada una de las partes y el todo) cobra especial importancia, ya que ésta crea un efecto amplificador de todas y cada una de las acciones realizadas por los involucrados.

La sinergia puede ser definida de diferentes formas, sin embargo todas estas definiciones convergen en una noción positiva en relación a los resultados obtenidos a partir de las interacciones entre los componentes de un sistema (Chadwick, 2010). Una definición aceptada de sinergia es la de Milgrom y Roberts (1992), que la definen como una circunstancia en la que se interrelacionan diversos individuos con iguales o diferentes capacidades; pero direccionadas bajo un mismo objetivo, permitiendo así la obtención de mayores beneficios dentro de una organización que de manera individual.

Cabe señalar que el desempeño de un sistema puede mejorar a partir de dos condiciones (las cuales no son mutuamente excluyentes): (1) La potenciación de los componentes individuales del sistema; y (2) La creación de interrelaciones eficaces y eficientes entre los componentes de un sistema. Dentro de este contexto es posible observar la sinergia desde tres perspectivas (Chadwick, 2010): (1) La perspectiva de superposición -dicha perspectiva asume que las prácticas pueden reforzar de manera mutua las interacciones (relaciones causales) entre los actores, dentro del sistema, para la obtención de esfuerzos direccionados en la obtención de más y mejores recursos y/o resultados-; (2) La perspectiva de efectos independientes -en este enfoque los efectos (beneficios y/o recursos) son independientes de la interacción entre los actores dentro del sistema, es decir, los beneficios obtenidos dentro de la organización, red, etc., son la resultante de habilidades individuales (sin una presencia de interacciones reales entre los entes) cuyos resultados, independientes, se van acumulando hasta alcanzar la meta propuesta-;y finalmente(3) La perspectiva de la eficiencia complementaria -bajo esta visión las interacciones prácticas y la especialización práctica de los actores (de manera balanceada), en donde el conjunto de prácticas son alineadas con otras, con el objeto de alcanzar un grado aceptable de efectos interactivos al explotar la independencia de forma recíproca entre los componentes del sistema-.

En la práctica, lo más importante dentro de un estudio social, en lo que a sinergia se refiere, es determinar el enfoque más adecuado, de acuerdo a la importancia relativa de las interacciones de sus componentes y la especialización de éstos (Chadwick, 2010). Sin embargo, existen otros factores (contingentes) que influencian esta decisión: (Chadwick, 2010 y Evans; 1996): (1) El nivel potencial de desempeño y la composición de los recursos; (2) Los criterios de medición del desempeño; (3) La robustez y la heterogeneidad dentro de la red; (4) La flexibilidad ante condiciones de cambio; (5) La vulnerabilidad ante los 
efectos de sustitución; (6) La habilidad de coordinación del sistema; (7) La vulnerabilidad y las disinergias; (8) La complementariedad; (9) Los intereses sociales, políticos y económicos de los actores; y finalmente, (10) La integración o incorporación -donde prevalece la idea que el comportamiento de los actores se encuentra incorporado en las normas y las instituciones de un entorno dado-.

La sinergia, vista desde el punto de vista ya expuesto, no es más que una extensión de la conducta colectiva en donde se reconoce el rol del ambiente institucional, donde se ven envueltos los individuos y/o grupos sociales. En este ámbito, el capital social resulta ser la base de la cooperación e interrelación, entre y dentro de las comunidades, tendiente al intercambio y/o consecución de bienes/recursos/servicios mediante la construcción de sinergias para la consecución y la promoción del desarrollo social sostenible en pos del bienestar individual y social de las comunidades. Dichas sinergias, generalmente son establecidas hacia el interior y el exterior de la comunidad. Hacia el exterior, se observan las sinergias a través de la calidad de las relaciones interinstitucionales con el Estado, utilizando la sociedad civil como catalizador, facilitando el surgimiento de la confianza, la red social y la movilización social para fines de desarrollo comunitario. Lo anterior no sólo permite una interrelación de cooperación entre la sociedad civilcomunidad-Estado de manera tangible, sino que también origina el establecimiento de sinergias como soporte de las relaciones entre los actores basados en la confianza y en la complementariedad, integradas dentro de un sistema de normas que rigen el comportamiento interno y externo de los actores para la obtención de beneficios y/o recursos (Chadwick, 2010).

\subsection{Las organizaciones de la sociedad civil}

El capital social se manifiesta de diferentes maneras, y la más tangible, la forman las organizaciones de la sociedad civil (OSC). La sociedad civil es la composición de instituciones como iglesias, sindicatos, escuelas, etc., que dan sustento a la vida social dentro una sociedad, éstas a su vez describen la totalidad de las redes sociales existentes e instituciones que operan de manera independiente del Estado y de los mercados, las cuales poseen diferencias en la significancia de sus roles bien definidos dentro del quehacer social (Walker et al, 2005). La sociedad civil permite no solamente la asignación de roles bien definidos, sino que, de igual manera, genera la cohesión social necesaria para la conformación de una sociedad como fuente de cambio.

Dentro de esteámbito las organizaciones de la sociedad civil pueden clasificarse (Asian Development Bank, 2008) de acuerdo a: (1) La razón de ser de la organización (Cuadro 2); y (2) la función que desempeñan dentro de una sociedad (Cuadro 3).

\subsubsection{Las OSC's y los cambios en el sector público}

El rol de las OSC's en la entrega se servicios han sido objeto de grandes transformaciones a lo largo de las últimas décadas, tanto en países industrializados como en vías de desarrollo, debido a que a nivel internacional se ha estado instrumentado dos grandes cambios en el entorno político, que afectan la perspectiva desde la cual se observa la importancia y el trabajo las OSC (Clayton et al, 2000). 


\section{Cuadro 2}

\section{Tipos de Organizaciones de la Sociedad Civil}

\begin{tabular}{|c|c|}
\hline Tipo de organización & Descripción \\
\hline $\begin{array}{l}\text { Organizaciones basadas en } \\
\text { comunidades (OBC) }\end{array}$ & $\begin{array}{l}\text { Son generalmente organizaciones directamente relacionadas con los intereses, } \\
\text { preocupaciones o demandas de sus miembros. Una característica clave de las OBC es } \\
\text { que éstas movilizan a las comunidades mediante la expresión de la organización, la } \\
\text { implementación de procesos participativos, acceso a servicios de desarrollo externos } \\
\text { y comparten los beneficios entre los miembros de la organización. }\end{array}$ \\
\hline $\begin{array}{l}\text { Organizaciones no } \\
\text { gubernamentales (ONG) }\end{array}$ & $\begin{array}{l}\text { Se refiere a organizaciones profesionales, intermediarias y sin fines de lucro que } \\
\text { se avocan o proveen a la provisión de servicios en relación al desarrollo social y } \\
\text { económico, derechos humanos, beneficios sociales o de protección civil. }\end{array}$ \\
\hline ONG de desarrollo & $\begin{array}{l}\text { Este tipo de organizaciones se encuentran orientadas sobre todo a cuestiones sociales } \\
\text { y humanitarias relacionadas con el desarrollo individual y comunitario, para el beneficio } \\
\text { y bienestar social y reducción de pobreza. }\end{array}$ \\
\hline ONG internacionales & $\begin{array}{l}\text { Son todas aquellas organizaciones definidas por la resolución } 288(x) \text { del Consejo } \\
\text { Social y Económico de las Naciones Unidas, "cualquier organización internacional no } \\
\text { fundada en un tratado internacional" }\end{array}$ \\
\hline $\begin{array}{l}\text { Organizaciones basadas en } \\
\qquad \text { la fe }\end{array}$ & $\begin{array}{l}\text { Son grupos religiosos organizados alrededor de congregaciones, cultos, institutos } \\
\text { religiosos especializados o en instituciones registradas/no registradas con carácter } \\
\text { religioso. }\end{array}$ \\
\hline Fundaciones & $\begin{array}{l}\text { Son organizaciones filantrópicas o caritativas establecidas por individuos o } \\
\text { instituciones como entidades legales, en donde las causas que apoyan se encuentran } \\
\text { soportadas y justificadas en los objetivos y metas de la organización. }\end{array}$ \\
\hline Sindicatos & $\begin{array}{l}\text { Son asociaciones formalmente organizadas de trabajadores que se encuentran unidos } \\
\text { para la promoción de beneficios colectivos en materia de salarios, jornadas laborales } \\
\text { y condiciones de trabajo. }\end{array}$ \\
\hline $\begin{array}{l}\text { Organizaciones de } \\
\text { personas (OP) }\end{array}$ & $\begin{array}{l}\text { Son entidades que tiene como base organizaciones de voluntarios que favorecen el } \\
\text { bienestar social y económico de sus miembros, los cuales se encuentran inmersos en } \\
\text { sectores particulares de la sociedad. }\end{array}$ \\
\hline $\begin{array}{l}\text { Asociaciones de } \\
\text { profesionales }\end{array}$ & $\begin{array}{l}\text { Este tipo de organizaciones generalmente se encuentran conformadas por individuos } \\
\text { que desempeñan una ocupación o una profesión en particular. }\end{array}$ \\
\hline Institutos de investigación & $\begin{array}{l}\text { Dichas organizaciones desarrollan análisis e investigaciones en materia de política } \\
\text { pública, con la intención de diseminar los resultados y las recomendaciones obtenidas } \\
\text { con la esperanza de influenciar a los funcionarios encargados del proceso de la toma } \\
\text { de decisiones. }\end{array}$ \\
\hline $\begin{array}{l}\text { Movimientos } \\
\text { sociales }\end{array}$ & $\begin{array}{l}\text { Son grupos informales que intentan efectuar un cambio social a través de la } \\
\text { organización y acción colectiva. Estas organizaciones no son permanentes; sin } \\
\text { embargo, se organizan de forma rápida (debido a la coincidencia del objeto de } \\
\text { búsqueda) para conseguir sus objetivos y metas, y luego desaparecen. }\end{array}$ \\
\hline
\end{tabular}

Fuente: Adaptado de Asian Development Bank, 2008.

El primero de estos es un cambio en las políticas clave por los donantes, los cuales han sido y resultan ser los patrocinadores sobre los cuales se han apoyado y erogado los recursos 


\section{Cuadro 3}

\section{Clasificación de la OSC en términos de sus funciones}

\begin{tabular}{|c|c|}
\hline Tipo de organización & Descripción \\
\hline OSC de entrega & $\begin{array}{l}\text { Se ocupan principalmente de desarrollar, monitorear e implementar proyectos, } \\
\text { programas o servicios, y tienden a trabajar de manera muy cercana con las OBC, } \\
\text { generalmente trabajan en un nivel comunitario. }\end{array}$ \\
\hline OSC de representación & $\begin{array}{l}\text { Proveen una voz unificada para expresar preocupaciones y necesidades de grupos } \\
\text { particulares o ciudadanos. }\end{array}$ \\
\hline $\begin{array}{l}\text { OSC de construcción de } \\
\text { capacidades }\end{array}$ & $\begin{array}{l}\text { Este tipo de organizaciones dan soporte a otras OSC en lo referente a financiamiento, } \\
\text { capacitación y actividades de sensibilización y toma de conciencia. }\end{array}$ \\
\hline OSC de defensa y política & Proveen experiencia y cabildeo en relación a cuestiones y asuntos particulares. \\
\hline OSC de funciones sociales & Dichas organizaciones se dedican a fomentar actividades sociales de manera colectiva. \\
\hline OSC con otras funciones & $\begin{array}{l}\text { Organizaciones que se dedican al desarrollo de redes de trabajo dentro de grupos } \\
\text { marginados, o aquellas OSC que desarrollan múltiples funciones }\end{array}$ \\
\hline
\end{tabular}

Fuente: Adaptado de Asian Development Bank, 2008.

necesarios para la operación de las OSC dentro de los países desarrollados y en vías de transición. Es decir, que el principal motivo por el cual los donantes han apoyado a las OSC, es por la toma de conciencia sobre la carencia de sociedad civil fuerte (Clayton et al, 2000); una sociedad civil fortalecida permite demandar una mayor democracia y transparencia en las acciones gubernamentales (Robinson et al, 1997), en donde los ciudadanos tienden a exigir una mayor participación en la decisiones a corto, mediano y largo plazo.

Lo donantes, a su vez identifican que en la actualidad las OSC resultan ser un factor clave y estratégico en el fortalecimiento de la sociedad y de las interrelaciones sociales e institucionales a nivel macro y micro dentro de una región en particular, lo cual conlleva de una forma o de otra la obtención de beneficios/ recursos para el desarrollo del capital social y de las sinergias que favorecen en gran medida a sectores poblacionales que carecen de los recursos necesarios para tal fin.
El segundo de estos cambios es el surgimiento de un nuevo paradigma: La Nueva Administración Pública (NPM, por sus siglas en inglés), introducido por algunos países industrializados como El Reino Unido, Los Estados Unidos de Norteamérica y Nueva Zelanda; promovido recientemente por el Banco Mundial, en donde se plantea la contracción del gasto social y la transferencia de dichas responsabilidades al sector privado (Clayton et al, 2000). Las nuevas reformas emprendidas dentro del sector público en las décadas comprendidas entre 1980 y el año 2000, en países desarrollados y en vías de desarrollo, se encuentran orientadas bajo una perspectiva neoliberal, donde la reducción del papel de Estado y la privatización de los servicios públicos, son los principios sobre los cuales se fundamenta la NPM (Clayton et al, 2000). La NPM plantea la reducción de los altos presupuestos públicos, con el objeto de incrementar la provisión de los servicios públicos, incrementando el rol del sector privado mediante la creación de nuevas 
agencias, aumentando la competitividad interna y el desempeño de las instituciones responsables de los servicios en cuestión, lo cual implicaría desligar al Estado de su responsabilidad social, dando lugar a una privatización de los servicios públicos mediante la tercerización de la provisión. La tercerización de los servicios no resulta del todo conveniente, ya que el sector privado busca primordialmente la rentabilidad de los proyectos a través de la obtención de beneficios monetarios (primordialmente) de los clientes, y en contraste el Estado busca obtener beneficios intangibles y tangibles, pero para los usuarios y no necesariamente para el Estado mismo.

Es en este contexto, surgen las OSC como organizaciones capaces de asumir roles más activos (tercerización de servicios) dentro de la provisión de servicios, los cuales, por un motivo u otro, el Estado resulta incapaz de proveer. Sin embargo, existen diferentes tipos de relaciones entre el Estado y las OSC, las cuales se definen de acuerdo al contexto social imperante dentro de la sociedad. Por ejemplo, en países como la India y regiones como Latinoamérica las OSC funcionan más como apéndices o elementos asociados en la prestación de servicios públicos; en cambio, en países donde existen condiciones de emergencia o excepción, dichas organizaciones resultan ser las únicas proveedoras de servicios sociales. Sin embargo, cabe destacar que los servicios privados no necesariamente resultan ser mejores, tanto en costos como en desempeño (Clayton et al, 2000).

En concordancia con lo anterior, puede manifestarse que dimensionar un servicio público mediante la injerencia total del capital privado en sustitución del Estado, sin supervisión, sin control del costo, del servicio, sin posibilidad de inversión y revocación ni poder de decisión, puede conllevar a un desbalance geográfico dentro de la equidad social; ya que se estaría dejando de lado la diferenciación social que existe entre los usuarios, los intereses individuales y la razón de ser de los servicios públicos, provocando una marginalización de algunos sectores de la sociedad (Mackintosh, 1997). Ahora bien, si se considera que solamente puede haber una elección dentro de una gama de opciones cuando se cuenta con el capital económico necesario para ello, la subrogación o substitución de responsabilidad social del Estado, en el caso de los estratos sociales menos desfavorecidos, daría origen a una exclusión sistemática del bienestar social de aquellos individuos que no cuenten con los recursos necesarios como para acceder a los servicios sociales dentro de una región.

EnLatinoamérica, hoyen díalas OSCfuncionan como organizaciones intermediarias $\mathrm{y} / \mathrm{o}$ proveedoras de servicios y dependientes, en su mayoría, de los recursos públicos. Si bien es cierto que las OSC permiten, en algunos casos, implementar programas de desarrollo comunitario en puntos geográficos donde se le dificulta al Estado realizarlo, en la actualidad no existen estudios y evidencias concluyentes que siguieran que las OSC sean mucho más efectivas en llegar a los estratos más pobres mediante ayuda para el desarrollo social (Clayton et al, 2000) ni de proveer una mayor calidad del servicio a menor costo de forma sustentable y sostenible. Lo anterior no solamente conduce a preguntarse la forma en que se evalúan a las OSC en lo concerniente a su desempeño, sino también la manera en que contribuyen al desarrollo del capital social y en la construcción de sinergias para el desarrollo comunitario. 
Es por ello que en la actualidad se requiere no solamente evaluar el desempeño administrativo y contable de las OSC, sino que de igual forma y de manera paralela, se hace necesaria la evaluación y la medición del desempeño, impactos y beneficios, generados dentro del marco comunitario; de lo contrario, no podrán existir estadísticas confiables sobre la eficacia y la eficiencia de las OSC dentro del desarrollo social bajo un marco de transparencia institucional.

Los impactos sociales son las consecuencias de un grupo social (actores) como resultado de cualquier acción pública o privada, las cuales alteran la manera en que se interrelacionan los individuos dentro de un sistema social; dichas alternaciones pueden ser: biofísicas, económicas, sociales, políticas, legales, culturales y/o psicológicas. Estos cambios pueden ser mesurados de manera directa o indirecta mediante la conceptualización de indicadores bien establecidos (Walker et al, 2000), en donde el impacto de las nuevas políticas y cambios tecnológicos pueden provocar una caída en los ingresos, ocasionando una mayor tasa de desempleo (Talbot et al, 2007). En la actualidad existen diversas metodologías para la medición del impacto social; sin embargo, en la práctica no resulta ser sencilla la manera en que se deben de evaluar y medir los impactos sociales de manera expost.

\subsection{El impacto social}

La medición del impacto social (SIA, por sus siglas en inglés) es el proceso de medir y administrar las consecuencias de los proyectos de desarrollo, políticas y decisiones sobre los integrantes de un entorno dado (Momtaz, 2005). El objeto es identificar los efectos deseados y no deseados de una intervención planeada, en orden de desarrollar y administrar planes de manera sostenible. EI SIA emergió como una disciplina separada dentro del campo social a partir de la realización de El Protocolo Nacional para las Políticas Ambientales en 1970 en los Estados Unidos de Norteamérica (The National Environmental Policy Act, NEPA), el cual no se encontraba direccionado de forma adecuada hacia cuestiones sociales.

EI SIA resulta ser básicamente un componente integral de la medición del impacto ambiental (Environmental Impact Assesment, EIA), cuyo objeto es el estudio de los probables cambios en el ambiente físico y biológico, así como en entorno socioeconómico, (y sus respectivas interrelaciones) como resultante de la planeación e implementación de un proyecto o una acción en particular (Momtaz, 2005).

Dentro de los componentes de la lista de verificación ambiental, el elemento humano incluye, entre otros campos, el de la salud y la seguridad, lo social, lo económico, lo cultural y lo estético. Caber mencionar que dentro del EIA el aspecto social cobra especial importancia dentro del procedimiento de medición de impactos desde que la perspectiva que los individuos dentro de una sociedad son los sujetos afectados o beneficiados dentro del ámbito económico y ambiental (Ahmadvand et al, 2009). De igual manera dentro del EIA se han realizado algunas simplificaciones como la matriz simplificada de medición de impactos (Rapid Impact Assessment Matrix, RIAM). Dicha matriz se desarrolló con el propósito de realizar de una manera transparente los criterios subjetivos dentro de la EIA, donde se evalúan impactos 
ambientales, sociales y socioeconómicos (ljäs et al, 2010).

Los objetivos del SIAgeneralmente tienden a ser directos, mediante la identificación de los impactos de forma prospectiva, con la finalidad de mejorar el proceso de toma de decisiones, así como de búsqueda de mitigación y de compensación para la minimización de impactos no deseados derivados del proyecto mismo (Tilt et al, 2009). Es por lo anterior, y respondiendo a las necesidades internacionales y locales para el SIA, la Asociación Internacional para la Medición de Impactos (IAIA, por sus siglas en inglés) desarrolló un conjunto de directrices aplicables a una amplia gama de tipos de proyectos (Tilt et al, 2009). Dichos principios incluyen entre algunas otras cosas, principios precautorios, igualdad intra e intergeneracional, la preservación social de la diversidad cultural y la internalización de los costos asociados con la planeación y la ejecución de los proyectos.

El problema de la medición del impacto social comienza desde la determinación de la envergadura del estudio. En este sentido se pueden identificar al menos tres tipos de impacto social susceptible de medición: (1) Impactos sociales a nivel micro -analiza impactos sobre el comportamiento de un número grande de individuos, como impactos demográficos-; (2) Impactos sociales a nivel meso -analiza los impactos sobre el comportamiento de actores colectivos como organizaciones y movimientos sociales- ; y (3) Impactos sociales a nivel macro -analiza impactos sobre macro sistemas, como pueden ser sistemas internacionales, nacionales, legales, etc. -.
Una vez identificados los tipos de impacto social se pueden identificar dos fases de medición (y sus respectivas actividades): (1) La fase inicial para la medición del impacto social de un proyecto -la cual incluye el análisis del problema y la comunicación de la estrategia, el análisis del sistema, el análisis de tendencias, el monitoreo del diseño y el diseño del proyecto; y (2) La fase principal para la medición del impacto social de un proyecto -la cual incluye el diseño de escenarios, el diseño de estrategias, la medición de impactos, la jerarquización de estrategias, la mitigación de impactos negativos, el reporte, estimulación de la implementación y la auditoría y evaluación expost. Cabe destacar que los estudios para la evaluación del impacto social (SIA) requieren de una medición asertiva del riesgo inmerso dentro del proyecto, de manera efectiva, considerando los procesos demográficos, económicos, geográficos, institucionales y legales, socioculturales, entre otros (Vanclay, 2002), considerando que el desarrollo es por naturaleza social, y dentro de éste se encuentran embebidos los valores sociales en curso, es decir, dentro del proceso social y las instituciones en donde los beneficios y costos derivados del desarrollo social son distribuidos a través de las comunidades, grupos sociales y organizaciones (Baker, 2000).

Dentro de los esfuerzos por medir el impacto social de proyectos y programas el Banco Mundial (BM), no prescribe algún procedimiento, técnica o metodología en específico; más bien, el BM plantea que el método deberá ser escogido de acuerdo a los objetivos de la medición del proyecto, el contexto social y los recursos y el tiempo disponible para tal fin. Cabe señalar que básicamente el BM propone cuatro tipos 
diversos de metodologías, como son (Francis et al, 1999):

1. Los diseños de experimentos: los diseños experimentales son las metodologías más sólidas de evaluación. Al distribuir aleatoriamente la intervención entre los beneficiarios calificados, el proceso de asignación crea grupos de tratamiento y de control comparables y estadísticamente equivalentes entre sí, a condición de que las muestras sean de tamaño adecuado.

2. Los diseños cuasiexperimentales: este tipo de técnicas generan grupos de comparación que se asemejan al grupo de tratamiento, al menos en las caracter ísticas observadas, usando metodologías econométricas que incluyen métodos de pareo, métodos de doble diferencia, métodos de variables instrumentales y comparaciones reflexivas. Cuando se usan estas técnicas, los grupos de tratamiento y de comparación por lo general se seleccionan después de la intervención usando métodos no aleatorios.

3. Los enfoques sin escenario contrafactual: son estudios cualitativos que evalúan las condiciones de la población (a menudo mediante la identificación de subgrupos vulnerables) antes, durante y después de las políticas de ajuste y se implementan a través de grupos representativos, entrevistas y otras técnicas cualitativas.

4. Los enfoques que generan un escenario contrafactual usando múltiples supuestos: Los modelos computarizados de equilibrio general (CGE) intentan contrastar los resultados en los grupos de tratamiento y de comparación mediante simulaciones. Estos modelos pretenden rastrear el funcionamiento de la economía real, y en general se basan en matrices de contabilidad social detalladas que se recopilan de datos sobre cuentas nacionales, encuestas de gastos familiares y otros datos de encuestas.

Bajo la temática de la medición del impacto social existen en la actualidad diversas técnicas y métodos; sin embargo, también existen otros dos enfoques capaces de evaluar el impacto social de una manera efectiva, a través de la utilización de técnicas participativas de planeación entre las cuales se tienen:

1. Los métodos multicriterio aplicados a la medición de impactos sociales. Las técnicas multicriterio son aquellas que analizan diversas alternativas de solución mediante criterios múltiples a través de relaciones binarias de sobreclasificación, aunque existen otras técnicas como las sumas ponderadas o el proceso de jerarquización analítica. Las técnicas multicriterio se basan en la comparación de parejas de soluciones, utilizando información cuantitativa y/o cualitativa para evaluar simultáneamente un conjunto de alternativas bajo diversos criterios de evaluación, los cuales pueden ser homogéneos, heterogéneos, cuantificables, cualificables o una mezcla de éstos (Gamboa, 2006; CloquellBallester et al., 2006). La evaluación social multicriterio (Social Multi-Criteria Evaluation, SMCE) es un marco de referencia sobre el cual es posible integrar la evaluación de las decisiones políticas, considerando de manera simultánea las dimensiones socioeconómicas, 
ecológicas, culturales, políticas y técnicas en la realización o ejecución de un proyecto de desarrollo social bajo cuatro etapas (Gamboa, 2006): (1) Análisis histórico e institucional (identificación de actores sociales y definición del problema); (2) Encuestas y entrevistas y grupos focales (establecimiento de criterios de evaluación y elicitación y la creación de alternativas y definición de los criterios de evaluación); (3) Trabajo multiinterdisciplinario (selección de la técnica multicriterio a utilizar y aplicación del modelo); y (4) Proceso participativo (análisis de los resultados por parte de los actores sociales).

2. Los foros comunitarios interactivos: Los foros comunitarios interactivos (Interactive Community Forum, ICF) fueron desarrollados por el Cuerpo de Ingenieros de la Armada de Los Estados Unidos de Norteamérica a partir de estudios de impacto ambiental (Environmental Impact Sudy, EIS). Los ICF son una metodología participativa para la medición de impactos sociales, en donde se ven involucrados los ciudadanos para prever los posibles impactos de un proyecto, de manera anticipada y de las posibles alternativas de solución. Dicha metodología representa una manera interdisciplinaria a partir del SIA, que conjunta los desarrollos teóricos y los estudios empíricos a partir de los campos socio-psicológico, psicología social, aprendizaje colaborativo, participación ciudadana y administración del ambiente, en donde la implementación de la metodología se encuentra dada por (Becker et al, 2003): (1) Selección de las comunidades; (2) Selección de los miembros de la comunidad; (3) Implementación de técnicas para pequeños grupos para el aprendizaje mutuo; (4) Identificación del rol de la información suplementaria; (5) Facilitación de talleres de trabajo; y (6) Identificación de las dimensiones y características (sociales, políticas, económicas, etc.), de la comunidad, para la identificación y la evaluación de impactos.

Entonces, con base a lo anterior resulta válido mencionar que más que presentar un enfoque rector sobre el capital social y la sinergia, dichos conceptos deben entenderse desde una óptica diferente, en donde la identificación de las variables de interés, involucradas en la medición del impacto social generado a través de los programas de coinversión social 1, dependerán de las características y la envergadura del estudio (micro, meso y macro) y del objetivo meta que se trace, es decir, lo que determina el concepto (sinergia y capital social) no es un autor en particular, más bien, es el enfoque que se requiera implementar. Lo anterior permite, entre otras cosas, flexibilizar la forma de medición, con el fin de mesurar, de forma adecuada, de una u otra manera, todas aquellas variables y bienes intangibles de la mejor manera posible, manteniendo en mente si las interrelaciones de interés son a nivel individual, grupal o colectivas a gran escala.

\section{Conclusiones}

En la actualidad, en El Salvador, no existe ningún mecanismo bien definido para la valoración y la visibilidad del trabajo desarrollado por las organizaciones de la sociedad civil, es decir, la inexistencia de una 
metodología adecuada para la valoración del capital social construido a través de las OSC, mediante el uso de indicadores confiables, origina una falta de visualización adecuada de todos aquellos aspectos que se encuentran más allá de los beneficios materiales, dando a lugar una visión parcial de los esfuerzos realizados por dichas organizaciones, así como la eficiencia y la eficacia en la aplicación de los recursos por parte de éstas en el desarrollo de proyectos sociales de coinversión social.

Dentro del concepto del capital social, podrían aprovecharse las sinergias actuales y existentes (y todos los componentes que conforman el capital social) entre los diversos grupos sociales, con el propósito de implementar proyectos de coinversión social, es decir, aprovechar las capacidades de una red social dada, para que el Estado pueda invertir en proyectos sociales utilizando la capacidad instalada de las OSC y el tejido social existente (municipio, colonia, sector, etc.), lo cual crearía un sentido de apropiabilidad, pertenencia y empoderamiento hacia el interior de la red social, generado de manera derivativa ahorros sustanciales en la ejecución de proyectos, dando origen a una nueva forma de desarrollo comunitario dentro de los estratos más vulnerables.

Y finalmente, la implementación de un proyecto social lleva consigo la responsabilidad de medir el impacto expost, con el objeto de medir la forma en que los beneficios derivados del proyecto han permeado a través del tejido social, además de cuantificar el incremento del bienestar (de manera cualitativa y cuantitativa) de la población objetivo; todo ello con miras de observar en retrospectiva la forma en que se hallevado a cabo el proyecto, la medición de beneficios y las áreas de mejoras (para futuros proyectos), para construir un marco comparativo unificado sobre el éxito o fracaso de los proyectos sociales.

\footnotetext{
1 Los programas de coinversión social (PCS) buscan la creación de capital social y el fortalecimiento institucional, promoviendo y fortaleciendo la participación de la sociedad civil organizada en acciones de desarrollo social que beneficien a personas en situación de pobreza y/o vulnerabilidad (Verduzco et al, 2009 e Indesol, 2015)
}

Artículo recibido: 7 de mayo de 2014 Artículo aprobado: 4 de julio 2014 


\section{Bibliografía}

ADGER, W., 2003, Social capital, collective action, and adaptation to climate change, Economic Geography 79 (4): 387-404.

AHMADVAND M, KARAMI E., HOSSEIN G., y VANCLAY, F. 2009, Evaluating the use of Social Impact Assessment in the context of agricultural development projects in Iran, Environmental Impact Assessment Review 29:399-407.

ASIAN DEVELOPMENT BANK, 2008, Civil Society Organization Sourcebook: A Staff Guide to Cooperation with Civil Society Organizations, Mandaluyong City, Philippines.

BAKER J., 2000, Evaluación del impacto de los proyectos de desarrollo en la pobreza, Manual para profesionales, Banco Internacional de Reconstrucción y Fomento/Banco Mundial 1818 H Street, N.W. Washington, D.C. 20433

BOURDIEU, P., 1986, The forms of capital. In: Richardson, J. (Ed.), Handbook of Theory and Research for the Sociology of Education. Greenwood Press, Westport, CT: 241-258.

BAKER J., 2000, Evaluación del impacto de los proyectos de desarrollo en la pobreza, Manual para profesionales, Banco Internacional de Reconstrucción y Fomento/Banco Mundial 1818 H Street, N.W. Washington, D.C. 20433

BECKER D., HARRIS C., MCLAUGHLIN W. y NIELSEN E., 2003, A participatory approach to social impact assessment: the interactive community forum, Environmental Impact Assessment Review 23, 367-382

BECKER H., 2001, Social impact assessment, European Journal of Operational Research 128, 311-321

BRUNIE A., 2009, Meaningful distinctions within a concept: Relational, collective, and generalized social capital, Social Science Research 38: 251-265.

CARPIANO R., 2006, Toward a neighborhood resource-based theory of social capital for health: Can Bourdieu and sociology help? Social Science \& Medicine 62: 165-175.

CHADWICK C., 2010, Theoretic insights on the nature of performance synergies in human resource systems: Toward greater precision, Human Resource Management Review 20: 85-101

CLARIDGE, T., 2007, Social capital, Retrieved October 14, <disponible en: http://www. gnudung.com/intro.html>.

CLAYTON A., OAKLEY P. y TAYLOR J., 2000, Civil Society Organizations and Service Provision, United Nations Research Institute for Social Development Civil Society and Social Movements Programme, Paper Number 2 October.

CLOQUELL-BALLESTER V., CLOQUELLBALLESTER V., MONTERDE-DÍAZ R, SANTAMARINA-SIURANA M., 2006, Indicators validation for the improvement of environmental and social impact quantitative assessment, Environmental Impact Assessment Review 26: 79- 105 
COLEMAN, J., 1988, Social capital in the creation of human capital. American Journal of Sociology 94 (Supplement): 95-120.

COLEMAN, J., 1990, The foundations of social theory. Cambridge, MA: Belknap.

EVANS, P., 1996, Government action, social capital, and development: reviewing the evidence on synergy. World Development, 24: 1119-1132.

FIELD, J., 2003, Social Capital, Routledge, New York.

FRANCIS P. Y JACOBS S., 1999, Institutionalizing Social Analysis at The World Bank, Environmental Impact Assessment Review 19: 341-357.

FORNI P., SILES M. Y BARREIRO L., 2004, ¿Qué es el Capital Social y cómo Analizarlo en contextos de Exclusión Social y Pobreza? Estudios de Caso en Buenos Aires, Argentina, Julian Samora Research Institute, Michigan State University, Research Report No. 35 December.

GAMBOA, G., 2006, Social multi-criteria evaluation of different development scenarios of the Aysén region, Chile, ECOLOGICAL ECONOMICS 59: 157-170

HOOGHE, M., 2008, Voluntary associations and socialization. In: Castiglione, D., van Deth, J., Wolleb, G. (Eds.), The Handbook of Social Capital. Oxford University Press, Oxford: 568-593.

HURLBER J., HAINE V. y BEGGS J., 2000, Core networks and tie activation: What kind of routine networks allocate resources in nonroutinesituations? American Sociological Review 65 (4): 598-618.

IJÄS A., KUITUNEN M. y JALAVA K., 2010, Developing the RIAM method (rapid impact assessment matrix) in the context of impact significance assessment, Environmental Impact Assessment Review 30 (2010) 82-89.

Indesol. 2015. COINVERSIÓN SOCIAL, SEDESOL, indesol.gob.mx/programas/ coinversión-social/

KRISHNA, A., 2002, Active Social Capital: Tracing the Roots of Development and Democracy. Columbia University Press, New York.

KRISHNA, A., 2008, Social capital and economic development. In: Castiglione, D., van Deth, J., Wolleb, G. (Eds.), The Handbook of Social Capital. Oxford University Press, Oxford: 438-466.

LIN, N., 2008, A network theory of social capital. In: Castiglione, D., van Deth, J., Wolleb, G. (Eds.), The Handbook of Social Capital. Oxford University Press, Oxford: 50-69.

MACKINTOSH, M., 1997, Public Management for Social Inclusion, paper presented at the conference on Public Sector Management for the Next Century, Manchester, 29 June - 3 July.

MILGROM, P., y ROBERTS, J., 1992, Economics, Organization, and Management. Englewood Cliffs, NJ: Prentice-Hall. 
MOMTAZ S., 2005, Institutionalizing social impact assessment in Bangladesh resource management: limitations and opportunities, Environmental Impact Assessment Review 25: 33-45.

MURPHY, B., FLAKINER L., MCBEAN G. and DOLLANT H. and Kovacs P., 2005, Enhancing local level emergency management: The influence of disaster experience and the role of households and neighborhoods. Paper series 43. Toronto: Institute for Catastrophic Loss Reduction, Retrieved January 10, 2007. <disponible en: http://www.iclr.org/pdf/ pine\%201ake\%2obrenda\%2omurphy.pdf $>$.

NEWTON, K., 1997, Social capital and democracy. American Behavioral Scientist 40 (5): 575-586.

OSTROM, E., 2000, Social capital: Fad or a fundamental concept? In: Dasgupta, P., Serageldin, I. (Eds.), Social Capital, a Multifaceted Perspective. The World Bank, Washington, DC: 172-214.

PORTES, A., 1998, Social capital: Its origins and applications in modern sociology, Annual Review of Sociology 24 (1): 1-24.

PUTNAM, R., 1993, The prosperous community: Social capital and public life. American Prospect, 13: 35-42.

PUTNAM, R. D., 1995, Bowling alone: America's declining social capital. Journal of Democracy, 6: 65-78.

PUTNAM, R. D., 2000, Bowling alone: The collapse and revival of American community, New York: Simon and Schuster.
PUTNAM, R., 2001, Foreword. In S. Saegert, J. P. Thompson, \& M. R. Warren (Eds.), Social capital and poor communities (pp. xv-xvi), New York: Russell Sage Foundation.

ROBINSON, M. Y WHITE G., 1997, The Role of Civic Organizations in the Provision of Social Services: Towards Synergy, World Institute for Development Economics Research, Helsinki <disponible en: http:// www.wider.unu.edu/publications/ working-papers/previous/en_GB/rfa-37/>.

ROTHSTEIN B., y DIETLIND S., 2008, Political institutions and generalized trust. In: Castiglione, D., van Deth, J., Wolleb, G. (Eds.), The Handbook of Social Capital. Oxford University Press, Oxford: 273-302.

SHAH, D., 1998, Civic engagement, interpersonal trust, and television use: An individual-level assessment of social capital, Journal of the International Society of Political Psychology 19 (3): 469-496.

TALBOT L. y WALKER R., 2007, Community perspectives on the impact of policy change on linking social capital in a rural community, Health \& Place 13: 482-492.

TILT B., BRAUN Y. y DAMING H., 2009, Social impacts of large dam projects: A comparison of international case studies and implications for best practice, Journal of Environmental Management 90: S249-S257.

USLANER, E., 2003, Trust, democracy and governance. Can government policies influence generalized trust? In: Hooghe, M., Stolle, D. (Eds.), Generating Social 
Capital: Civil Society and Institutions in Comparative Perspective. Palgrave MacMillan, New York: 171-190.

VANCLAY F., 2002, Conceptualising social impacts, Environmental Impact Assessment Review 22: 183-211.

VAN DER GAAG, M., SNIJDERS T. y FLAP H., 2004, Position Generator measures and their relationship to other social capital measures. Working paper. Amsterdam, Vrije Universiteit, Retrieved October 4, 2005 <disponible en: http://www.xs4all. nl/gaag/work/PG_comparison.pdf $>$.

Verduzco M., Trujillo J., Tapia M., FONDOS PÚBLICOS PARA LAS ORGANIZACIONES DE LA SOCIEDAD CIVIL. ANÁLISIS DEL PROGRAMA DE COINVERSIÓN SOCIAL, Alternativas y Capacidades AC, México DF.

WAKEFIELD S. y POLAND B., 2005, Family, friend or foe? Critical reflections on the relevance and role of social capital in health promotion and community development, Social Science \& Medicine 60: 2819-2832.

WALKER D. y DEARDEN A. 2005, Editorial: Designing for civil society, Interacting with Computers 17: 1-8.

WALKER J., MITCHELL B. y WISMER S., 2000, Environmental Impact Assessment Review20: 13-535.

ZHAO, Yandong, 2002, Measuring the social capital of laid-off Chinese workers. Current Sociology 50 (7): 555-571. 